\begin{tabular}{|c|c|}
\hline Title & $\begin{array}{l}\text { A single-photon sensitive synchroscan streak camera for room temperature picosecond emission dynamics of adenine } \\
\text { and poly adenylic acid }\end{array}$ \\
\hline Author(s) & Kobay ashi, Shunsuke; Y amashita, Mikio; Sato, Takuzo; Muramatsu, Shigeru \\
\hline Citation & $\begin{array}{l}\text { IEEE Journal of Quantum Electronics, 20(12), 1383-1385 } \\
\text { https://doi.org/10.1109/JQ E.1984.1072335 }\end{array}$ \\
\hline Issue Date & 198412 \\
\hline Doc URL & http:/hdl.handle.net/2115/45319 \\
\hline Rights & $\begin{array}{l}\text { (C) } 1984 \text { IEEE. Personal use of this material is permitted. However, permission to reprint/republish this material for } \\
\text { advertising or promotional purposes or for creating new collective works for resale or redistribution to servers or lists, } \\
\text { or to reuse any copyrighted component of this work in other works must be obtained from the IEEE. }\end{array}$ \\
\hline Type & article \\
\hline File Information & JQE20-12_1383-1385.pdf \\
\hline
\end{tabular}

Instructions for use 


\title{
A Single-Photon Sensitive Synchroscan Streak Camera for Room Temperature Picosecond Emission Dynamics of Adenine and Polyadenylic Acid
}

\author{
SHUNSUKE KOBAYASHI, MIKIO YAMASHITA, TAKUZO SATO, AND SHIGERU MURAMATSU
}

\begin{abstract}
It is experimentally verified that a synchroscan streak camera, incorporating a microchannel plate and synchronizing with UV picosecond pulses generated inside the cavity of a mode-locked $\mathrm{CW}$ ring dye laser, has enough sensitivity to detect picosecond emission phenomena in the region of a single-photon event. To demonstrate the usefulness of the synchroscan streak camera, the first measurement of time- and wavelength-resolved emissions in the picosecond region is successfully carried out for adenine and polyadenylic acid aqueous solutions at room temperature, and shows that their shortest lifetimes, due to the monomer fluorescence, are 6 and 8 ps, respectively.
\end{abstract}

$\mathrm{F}$ OR a study on nucleic acid photochemistry and photobiology, it is essential to make clear the dynamic behavior of the luminescence from nucleic acid bases in mononers, polymers, and higher order structures. Many investigations of the excited state dynamics of nucleic acids and their components have been carried out [1]. However, their emissions at room temperature remain incompletely characterized because of their low quantum yields $\left(10^{-4} \sim 10^{-5}\right)$ and fast lifetimes $\left(10^{-11} \sim 10^{-13}\right.$ s) [2]. Room temperature emission spectra, quantum yields, and lifetimes of nucleotides were reported recently by Ballini $e t$ al. [3] and Rigler et al. [4] with a singlephoton counting method. They found that the emission quantum yields of adenine and polyadenylic acid were $2.4 \times 10^{-4}$ and $3 \times 10^{-4}$, respectively [3]. The lifetime of polyadenylic acid depended on the wavelength of the emission band, and the origin of more than three emission components was discussed [3]. However, the questions still remain because of the instrumental restriction of the time resolution. Therefore, further investigation based on time- and wavelength-resolved emission measurements in the picosecond time region is strongly needed.

An electron-optical streak camera in synchronous operation with a mode-locked CW dye laser has become the most useful instrument that can directly measure the time and wavelength versus the intensity profile of a picosecond optical event for the weak luminescence [5], [6]. Using a highly-sensitive synchroscan streak camera with a mode-locked and internally frequency-doubled $\mathrm{CW}$ ring dye laser, we report the first direct

Manuscript received August 1, 1984; revised September 10, 1984.

S. Kobayashi, M. Yamashita, and T. Sato are with the Laser Research Section, Radio- and Opto-Electronics Division, Electrotechnical Laboratory, Ibaraki 305, Tokyo, Japan.

S. Muramatsu is with the Department of Synthetic Chemistry, Faculty of Engineering, Kyoto University, Kyoto 606, Japan.

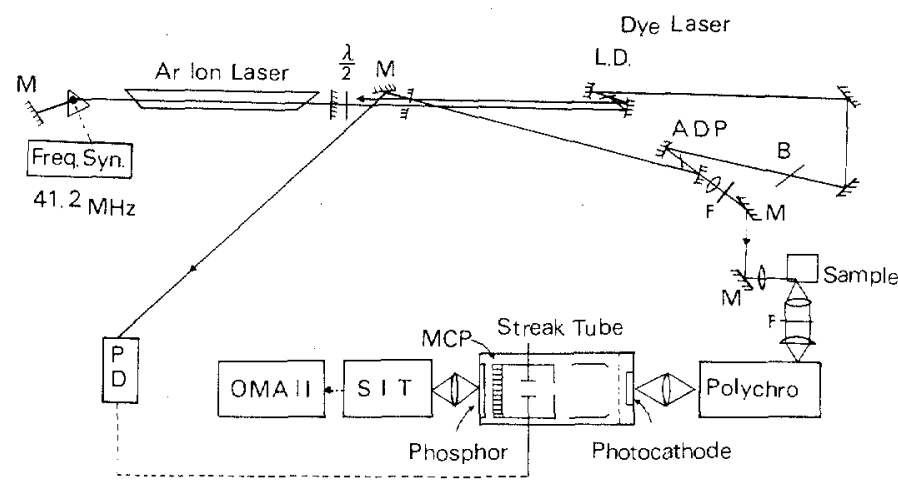

Fig. 1. Schematic diagram of an experimental arrangement for a picosecond weak emission measurement using a single-photon sensitive synchroscan streak camera with a mode-locked and internally frequency doubled $\mathrm{CW}$ ring dye laser; $\mathrm{M}:$ mirror, $\lambda / 2$ : half-wave plate, L.D.: laser dye, ADP: ADP crystal, B: quartz plate, F: filter, and PD: $p-i-n$ photodiode.

measurement of picosecond time- and wavelength-resolved emissions from adenine and polyadenylic acid in aqueous solutions at room temperature.

The experimental configuration used is shown schematically in Fig. 1, which is essentially similar to that previously reported [5], [7] except for the following points. An actively modelocked Ar ion laser (Spectra-Physics 171) was used to synchronously pump a Rhodamine $6 \mathrm{G}$ ring dye laser at the repetition rate of $82 \mathrm{MHz}$. A high long-term stability of the mode-locked Ar ion laser enabled us to produce continuous trains of $85 \mathrm{ps}$ pulses. A ring synchronous mode locking was chosen for efficient intracavity second-harmonic generation. Continuous trains of UV picasecond pulses at $291 \mathrm{~nm}$ had the average power of $0.2 \mathrm{~mW}$ per each direction under the pumping power of $1.7 \mathrm{~W}$.

The two-dimensional synchroscan streak camera (Hamamatsu Photonics C1587) in our system had a high sensitivity since a microchannel plate (MCP; gain $5 \times 10^{3}$ at $900 \mathrm{~V}$ ) was incorporated [8]. The streak image was monitored by a SIT vidicon of OMA II (Princeton Applied Research 1215/1216) which displayed and analyzed the two dimensional signal of timeresolved spectra or wavelength-resolved decays. When MCP is operated at $700 \mathrm{~V}$ to hold the reasonable signal-to-noise ratio, the streak camera amplifies one photon at the photocathode to $10^{3}$ photons at the phospher screen. Two percent of these are collected into the SIT cathode and recorded as one count 
by OMA II. Therefore, it is deduced that the sensitivity of the present synchroscan system is in the region of a single-photon event. Moreover, the photon utility of the streak camera at each event is far better than that of the time-correlated singlephoton counter.

The sensitivity was confirmed by the measurement of the very weak fluorescence from psoralen in ethanol. The UV picosecond pulses used to excite the psoralen solution was focused into a $10 \mathrm{~mm}$ square quartz sample cell. The fluorescence was collected at $90^{\circ}$ with respect to the incident beam and was focused into the entrance slit of a grating polychromator (Jobin Yvon HR 320). A pyrex plate was used to reduce light scattered from excitation UV pulses. The polychromator has a dispersion of $10 \mathrm{~nm} / \mathrm{mm}$ and covers the $120 \mathrm{~nm}$ spectral width. The results showed that even for the $7.2 \times 10^{-8} \mathrm{M}$ psoralen solution, time-resolved fluorescence spectra were observed at the $S / N$ of 15 in a few hundred picoseconds time region in the integration time of $15 \mathrm{~s}$. This means that the solution emits only 30 photons per pulse into all solid angles since the fluorescence quantum yield of psoralen is $0.8 \times 10^{-2}[9]$. The optical collection efficiency of the system is estimated to be $10^{-4}$. As a result, $3 \times 10^{-3}$ photons per pulse at the photocathode of the streak tube [8] are enough to measure timeresolved fluorescence spectra. Therefore, it is concluded that the sensitivity of the present two-dimensional synchroscan streak camera system is high enough to detect the emission in the region of a single-photon event. In addition, it was confirmed that the streak camera had a large dynamic range of $5 \times 10^{3}[8]$.

Synchronizing the emission signal with the deflection voltage of the streak camera was done by monitoring visible pulses from the dye laser with a p-i-n photodiode. The full width at half maximum (FWHM) of the UV pulses in the integration time of $1 \mathrm{~s}$ was $24 \mathrm{ps}$, while the FWHM was only 5 ps at the 100 ns gate time in which 8 pulses were accumulated. This broadening can be attributed to the arrival time jitter in the synchronously pumped dye laser system.

Adenine and polyadenylic acid (sodium salt) was purchased from Yamasa Shoyu Co., Ltd., and used without further purification. The water used for the fluorescence measurement was a Dotite Luminasole grade and no detectable emission was observed under the experimental conditions.

The time-resolved emission spectra measured for an adenine aqueous solution $\left(6 \times 10^{-4} \mathrm{M}\right)$ at room temperature are shown in Fig. 2. Each spectrum was accumulated in the successive 85 ps duration. A narrow Raman line from solvent $\mathrm{H}_{2} \mathrm{O}$ was observed at $320 \mathrm{~nm}$, in Fig. 2(a), and was used as a reference of the time profile of UV pulses because the Raman scattering is an instantaneous phenomenon and has the same optical geometry as fluorescence. A short wavelength emission, which corresponds to the reported monomer emission [3], was observed at $340 \mathrm{~nm}$ immediately after the excitation. The 340 $\mathrm{nm}$ emission disappeared within $42 \mathrm{ps}$, while a red shifted emission was predominant at $390 \mathrm{~nm}$ over several hundred picoseconds. The longer lifetime emission can be attributed to the adenine aggregate compared to the results for polyadenylic acid [3]. The corresponding wavelength-resolved decay curves are shown in Fig. 3 with the Raman scattering profile. The lifetime of the $340 \mathrm{~nm}$ emission was calculated to be 6 ps from the deconvolution of curve (a) measured as the response to

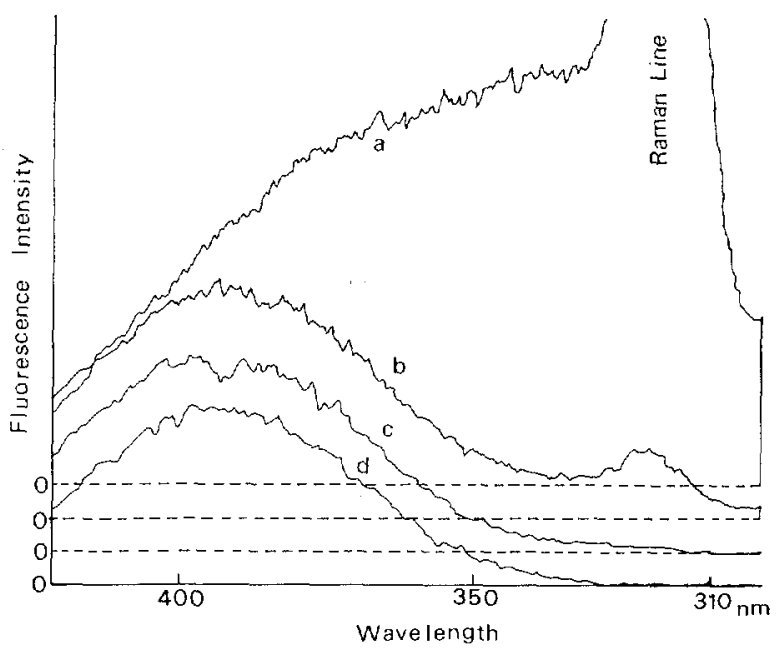

Fig. 2. Time-resolved emission spectra measured for an adenine aqueous solution $\left(6 \times 10^{-4} \mathrm{M}\right)$ at room temperature; (a) $0 \mathrm{ps,} \mathrm{(b)} 85 \mathrm{ps,} \mathrm{(c)}$ 170 ps, and (d) 340 ps.

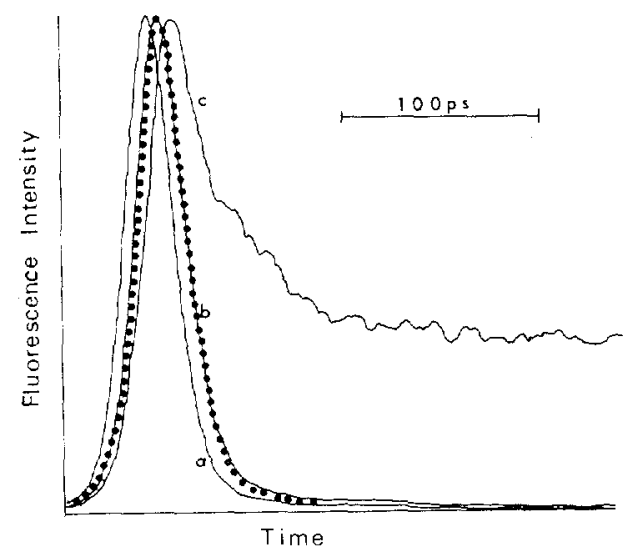

Fig. 3. Wavelength-resolved decay curves measured for an adenine aqueous solution $\left(6 \times 10^{-4} \mathrm{M}\right)$ at room temperature; (a) $320 \mathrm{~nm}$ (Raman scattering profile), (b) $340 \mathrm{~nm}$, and (c) $380 \mathrm{~nm}$. Closed circles represent the calculated value with the lifetime of $6 \mathrm{ps}$.

the excitation pulse and the curve (b) measured as the decay around the $340 \mathrm{~nm}$ emission [10]. The fitting was done within \pm 0.5 ps accuracy. The result is shown in Fig. 3 .

The temporal broadening $\Delta \tau(\lambda)$ due to the path difference for a monochromator at the given wavelength $\lambda$ was discussed by Schiller and Alfano [11] and estimated to be $\sim 10$ ps at $300 \mathrm{~nm}$ in our system. The pulse broadening inside the monochromator can be neglected, since 25 ps of the FWHM of Raman scattering, in Fig. 3(a), is in agreement with 24 ps of the FWHM of the laser pulse which is little affected by the width of the grating. Using $\Delta \tau(320 \mathrm{~nm})=10 \mathrm{ps}$ in our system and $\Delta \tau(340 \mathrm{~nm}) / \Delta \tau(320 \mathrm{~nm})=1.06$, one can estimate the difference between $\Delta \tau(320 \mathrm{~nm})$ of the reference wavelength and $\Delta \tau(340 \mathrm{~nm})$ of the monomer emission to be $0.6 \mathrm{ps}$. This can be neglected compared to the measured lifetime of $6 \mathrm{ps}$. The measured value is in agreement with the calculated lifetime of $1.0 \mathrm{ps}$ [2]. Therefore, the origin of the $340 \mathrm{~nm}$ emission is assigned to the lowest $\pi-\pi^{*}$ state. The lifetime of the $390 \mathrm{~nm}$ emission was measured to be 790 ps.

Fig. 4 shows the time-resolved emission spectra for a polyadenylic acid aqueous solution $\left(5.5 \times 10^{-4} \mathrm{M}\right)$. It should be noted that, unlike adenine, not only the $340 \mathrm{~nm}$ emission but also the $390 \mathrm{~nm}$ emission was observed in the shortest time region. The corresponding wavelength-resolved decay curves 


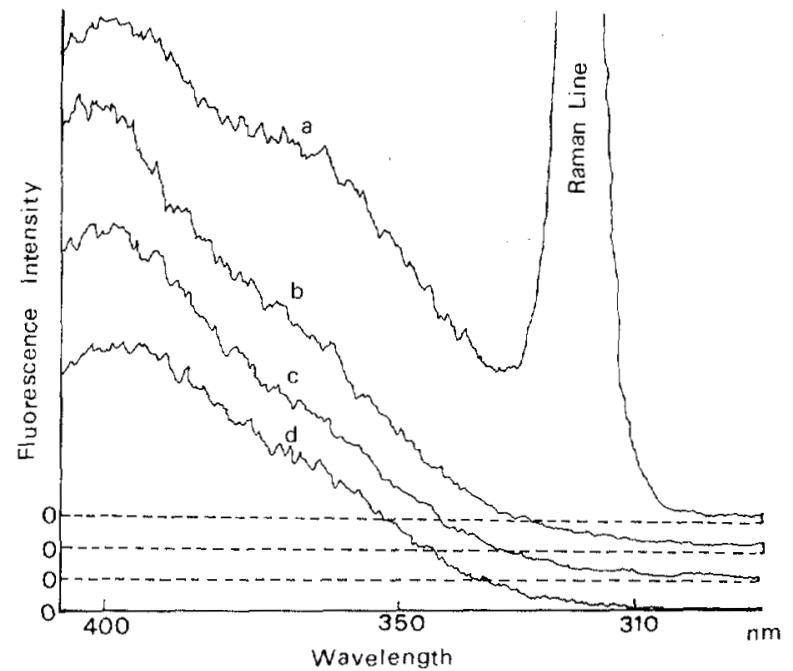

Fig. 4. Time-resolved emission spectra measured for a polyadenylic acid solution $\left(5.5 \times 10^{-4} \mathrm{M}\right)$ at room temperature; (a) $0 \mathrm{ps}$, (b) $85 \mathrm{ps}$, (c) $170 \mathrm{ps}$, and (d) $340 \mathrm{ps}$.

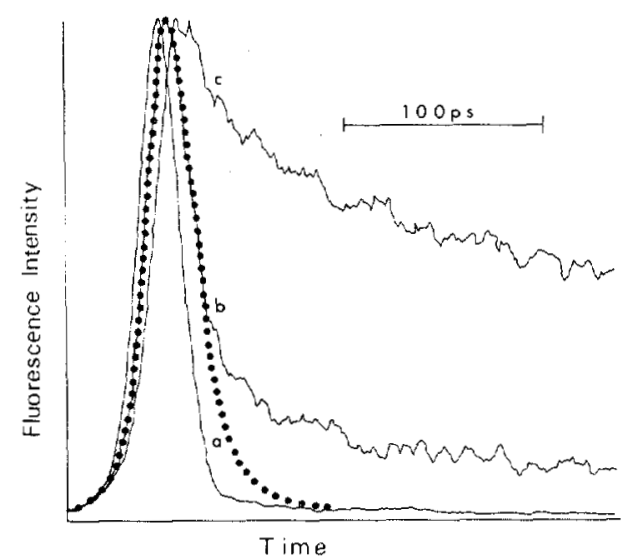

Fig. 5. Wavelength-resolved decay curves measured for a polyadenylic aqueous solution at room temperature; (a) $320 \mathrm{~nm}$ (Raman scattering profile), (b) $340 \mathrm{~nm}$, and (c) $380 \mathrm{~nm}$. Closed circles represent the calculated value with the lifetime of $8 \mathrm{ps}$.

are shown in Fig. 5. The short component of the decay curve shows a lifetime of 8 ps which is dominant at the $340 \mathrm{~nm}$ emission. The longer component of the decay curves has been fitted by two time constants of $510 \mathrm{ps}$ and $1.3 \mathrm{~ns}$, since it shows nonsingle exponential character. These may be assigned to the aggregate fluorescence of different configurations [3], [4], [12]. The radiative lifetimes of the excited states of aggregates estimated from the measured lifetimes and quantum yield are two-order-of-magnitude longer than those of monomers. The emitting states of the aggregates may be singlet with forbidden character. A possibility for the delayed excimer fluorescence, which was suggested by Ballini et al. [3] , is ruled out because no rise up at 340 and $390 \mathrm{~nm}$ was observed.

The red shifted $390 \mathrm{~nm}$ emission of adenine is similar to that found in polyadenylic acid, which was not reported by Ballini et al. [3]. The discrepancy may come from the different experimental conditions concerning the concentration and excitation wavelength. The further investigations including this point are in progress.

In summary, we have demonstrated that the two-dimensional synchroscan streak camera, incorporating the microchannel plate and operating synchronously with an efficient UV picosecond pulses at high repetition rates, is sensitive enough to detect the emission in the region of a single-photon event. Using this system, we have first measured picosecond timedependent emission spectra and wavelength-resolved decays at room temperature for adenine and polyadenylic acid aqueous solutions whose emissions have very low quantum yields and very short lifetimes.

\section{ACKNOWLEDGMENT}

The authors wish to thank Dr. M. Ishikawa, A. Hirano, and M. Nomura for their experimental assistance.

\section{REFERENCES}

[1] S. Y. Wang, Ed, Photochemistry and Photobiology of Nucleic Acids, vol. 1. New York: Academic, 1976, pp. 23-167, pp. $381-417$.

[2] M. Daniels, Photochemistry and Photobiology of Nucleic Acid. New York: Academic, 1976, pp. 23-108.

[3] J. P. Ballini, M. Daniels, and P. Vigny, "Wavelength-resolved lifetime measurements of emissions from DNA components and poly rA at room temperature excited with synchrotron radiation," J. Luminescence, vol. 27, pp. 389-400, 1982.

[4] R. Rigler, F. Claesens, and G. Lomakka, "Picosecond single photon fluorescence spectroscopy of nucleic acids," in Tech. Dig. Topical Meet. Ultrafast Phenomena, ThC3, Monterey, CA., June 12-15, 1984.

[5] M. Yamashita, S. Kobayashi, T. Sato, M. Nomura, and K. Aizawa, "Picosecond time-resolved fluorescence spectra of hematoporphyrins," in Tech. Dig. Topical Meet. Ultrafact Phenomena, ThE9, Monterey, CA, June 12-15, 1984.

[6] A. J. Campillo and S. L. Shapiro, "Picosecond streak camera fluorometry -A review," IEEE J. Quantum Electron., vol. QE-19, pp. 585-603, 1983.

[7] M. Yamashita, W. Sibbett, D. Welford, and D. J. Bradley, "Intracavity second-harmonic generation in a synchronously modelocked CW dye laser," J. Appl. Phys., vol. 51, pp. 3559-3562, 1980.

[8] Y. Tsuchiya, A. Takeshima, and E. Inuzuka, "Synchroscan streak camera," in 15th Int. Congr. High Speed Photography Photonics, Soc. Photo-opt. Instr. Eng., vol. 348, pp. 245-250, 1982.

[9] M. Ishikawa, "Relaxation mechanism of excited states of psoralen and its derivatives," Ph.D dissertation, Tohoku Univ., Sendai, Japan, 1984.

[10] A. Grinvald and I. Z. Steinberg, "On the analysis of fluorescence decay kinetics by the method of least-squares," Anal. Biochem., vol. 59 , pp. $583-598,1974$.

[11] N. H. Schiller and R. R. Alfano, "Picosecond characteristics of a spectrograph measured by a streak camera/video readout system," Opt. Commun., vol. 35, pp. 451-454, 1980.

[12] J. P. Ballini, P. Vigny, and M. Daniels, "Synchrotron excitation of DNA fluorescence decay time evidence for excimer emission at room temperature," Biophys. Chem., vol. 18, pp. 61-65, 1983.

Shunsuke Kobayashi, for a photograph and biography, see this issue, p. 1369.

Mikio Yamashita, for a photograph and biography, see this issue, p. 1369.

Takuzo Sato, for a photography and biography, see this issue, p. 1369.

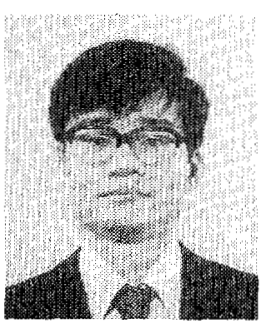

Shigeru Muramatsu was born in Osaka Prefecture, Japan, on January 12,1961 . He received the B.S. degree in synthetic chemistry from Kyoto University, Kyoto, Japan, in 1983.

He is now working toward the M.S. degree in photochemistry and photophysics of amino acids and nucleic acids derivatives at the Department of Synthetic Chemistry, Faculty of Engineering, Kyoto University.

Mr. Muramatsu is a member of the Chemical Society of Japan and the Society of Synthetic Organic Chemistry, Japan. 